\title{
Fluorescence in situ hybridization, a diagnostic aid in ambiguous melanocytic tumors: European study of 113 cases
}

Beatrice Vergier ${ }^{1}$, Martina Prochazkova-Carlotti ${ }^{1}$, Arnaud de la Fouchardière ${ }^{2}$, Lorenzo Cerroni ${ }^{3}$, Daniela Massi ${ }^{4}$, Vincenzo De Giorgi ${ }^{4}$, Christiane Bailly ${ }^{2}$, Ulrich Wesselmann ${ }^{5}$, Apollon Karlseladze ${ }^{6}$, Marie-Francoise Avril ${ }^{7}$, Thomas Jouary ${ }^{8}$ and Jean-Philippe Merlio ${ }^{1}$

${ }^{1}$ Département de Pathologie et Biologie des Tumeurs, EA2406, CHU Bordeaux-Université Victor Segalen Bordeaux 2, Hôpital Haut-Lévêque, CHU Pessac-Bordeaux, France; ${ }^{2}$ Département de Pathologie, Centre Léon Bérard (CLCC), Lyon, France; ${ }^{3}$ Department of Dermatology, Medical University of Graz, Graz, Austria; ${ }^{4}$ Department of Pathology and Oncology, University of Florence, Florence, Italy; ${ }^{5}$ Department of Dermatology, Helios Hospital Wuppertal, University of Witten-Herdecke, Witten-Herdecke, Germany; ${ }^{6}$ Department of Pathology, Russian Cancer Research Centre, Moscow, Russia; ${ }^{7}$ Service de Dermatologie, Hôpital Tarnier, Paris, France and ${ }^{8}$ Service de Dermatologie, Unité de Cancérologie Cutanée, Hôpital Saint André,

Bordeaux, France

Some melanocytic tumors are ambiguous, so the reproducible histopathological diagnosis of benign or malignant lesion is difficult. This study evaluated the contribution of fluorescence in situ hybridization (FISH) first in $\mathbf{4 3}$ non-equivocal melanomas and nevi, and then in 113 ambiguous melanocytic tumors selected by expert pathologists from six different European institutions. We included two groups of ambiguous tumors: patients without recurrence (5-year minimal follow-up) and with metastases. An independent triple-blind histopathological review was performed to classify tumors as 'favor benign' ( $\mathbf{A}-)$ or 'favor malignant' $(\mathbf{A}+)$. A four-color probe set targeting 6p25, 6q23, 11q13 and CEP6 was used for FISH. In the 43 non-equivocal melanomas and nevi, sensitivity was $85 \%$ and specificity $90 \%$. Ninety out of 95 ambiguous melanocytic tumors included were FISH interpretable (67 FISH negative and 23 FISH positive). Of the 90 patients, 69 presented no recurrence and 21/90 exhibited metastases. These ambiguous tumors were mostly spitzoid tumors (45/90). Histopathological reviewers classified these tumors as favor malignant (49/90) and favor benign (32/90), whereas nine cases had a discordant diagnosis. By comparison with outcome, the sensitivity and specificity of histopathological review were 95 and $\mathbf{5 2} \%$, and the sensitivity and specificity of FISH were 43 and $\mathbf{8 0} \%$. Compared with histopathological review, the sensitivity and specificity of FISH were 34.5 and $91 \%$. Interestingly, by combining the histopathological diagnosis with FISH results, the diagnosis was optimized, especially by increasing specificity ( $76 \%$ instead of $52 \%$ for expert diagnosis alone) and by improving sensitivity compared with FISH alone (90 vs $43 \%$ for FISH result alone). The value of this FISH test is to add a reproducible demonstration of malignancy to the histopathological diagnosis, especially in doubtful/ambiguous melanocytic tumors. A positive FISH test reinforces the diagnosis of melanoma, allowing such tumors (particularly thick tumors) to be managed as melanomas.

Modern Pathology (2011) 24, 613-623; doi:10.1038/modpathol.2010.228; published online 10 December 2010

Keywords: ambiguous melanocytic tumors; FISH; melanoma; MELTUMP; nevus; Spitz

Correspondence: Professor B Vergier, MD, PhD, Département de Pathologie et Biologie des Tumeurs, EA2406, CHU BordeauxUniversité Victor Segalen Bordeaux 2, Service de Pathologie, Hopital Haut-Lévêque, Avenue de Magellan, CHU PessacBordeaux 33604, France.

E-mail: beatrice.vergier@chu-bordeaux.fr

Received 16 April 2010; revised 4 October 2010; accepted 4

October 2010; published online 10 December 2010
Some melanocytic lesions are difficult to diagnose as truly benign or malignant and there is a subset of ambiguous cases in which a specific and reproducible diagnosis is difficult or even impossible to render. For problematic melanocytic lesions, a second opinion from an expert dermatopathologist 
improves patient care in $27 \%$ of cases. ${ }^{1}$ However, a complete agreement for difficult melanocytic neoplasms was observed only in $54.5 \%$ and a high level of disagreement (ie, benign vs malignant) was found in $25 \%$ of cases among consultant pathologists. ${ }^{1-5}$ Such ambiguous melanocytic tumors are divided into thin and thick tumors. ${ }^{4}$ Thin ambiguous melanocytic lesions are frequently encountered as dysplastic nevus or atypical intraepidermal melanocytic proliferation, thus raising the diagnostic issue of atypical nevus or truly superficial spreading thin or in situ melanoma. Owing to the lack of reproducible diagnosis, different names have been proposed, such as 'melanocytic intraepidermal neoplasia (MIN)' ${ }^{6,7}$ or 'superficial atypical melanocytic proliferations of uncertain significance (SAMPUS)' ${ }^{8}$ The prognosis of such thin atypical melanocytic tumors is excellent if they are completely excised, but a precise diagnosis remains difficult to make. This is not the case for 'thick' atypical melanocytic lesions, because the consequences for therapy and prognosis are more important. These thick ambiguous melanocytic lesions called 'melanocytic tumors of uncertain malignant potential (MELTUMP)' by Elder and $\mathrm{Xu}^{8}$ comprise melanocytic proliferations that form tumors in the dermis, and are therefore potentially capable of metastasis. The most frequent histological subtypes included in this category are Spitz tumors and melanoma simulating other types of nevus, deep penetrating nevus or cellular blue nevus. ${ }^{9}$ For both thick and thin ambiguous melanocytic lesions, new diagnostic and prognostic tools are necessary for second opinion and may improve diagnostic accuracy and reproducibility between expert dermatopathologists.

Very recently, Gerami et $a l^{10}$ used a multicolor fluorescence in situ hybridization (FISH)-based test as a new tool to provide the highest diagnostic discrimination between melanoma and nevus. It allows detection of copy number changes of chromosomal regions by the use of locus-specific probes developed on the basis of the presence of non-overlapping patterns of chromosomal imbalances in melanoma and nevi. ${ }^{11}$ This technique is especially applicable to melanocytic lesions as it can be performed on sections consecutive to those used for histopathological analysis.

The aims of our study were first to perform FISH analysis in 43 cases of non-equivocal melanomas (23) and nevi (20) in a training set. Then, we analyzed 113 cases of ambiguous melanocytic tumors provided by six European centers to determine the relevance of such testing by comparison with an expert panel review as well as with patients' clinical course. For this purpose, we selected ambiguous lesions in patients with at least 5 years recurrence-free follow-up and in patients with secondary regional metastasis (stage III) or distant metastasis (stage IV) or death.

\section{Materials and methods}

As a training set, we first included 43 patients with either non-equivocal melanomas (23 cases) or typical nevi (20 cases) to evaluate applicability or efficiency, sensitivity and specificity of the melanoma FISH test. All cases were provided by a single dermatopathological department (Bordeaux). Different histological subtypes of melanoma were included: superficial spreading melanomas (11 cases), lentigo malignant melanoma (three cases), acral lentiginous melanoma (three cases), unclassifiable melanomas (four cases) and desmoplastic melanoma (two cases). The different subtypes of nevi were common nevi (11 cases with one junctional nevus, five dermal nevi and five compound nevi), congenital nevi (four cases), common blue nevi (two cases), Spitz or Reed nevi (three cases) and genital type nevus (one case).

Secondly, we included 113 patients with ambiguous melanocytic tumors selected by expert pathologists in the field of melanoma working in six different European institutions: L Cerroni (Graz, Austria, 25 cases), D Massi (Firenze, Italy, 24 cases), U Wesselmann (Wuppertal, Germany, seven cases), A Karseladze (Moscow, Russia, seven cases), A de la Fouchardiere (Lyon, France, 14 cases) and B Vergier (Bordeaux, France, 36 cases). For this second step, two groups of patients with ambiguous/atypical melanocytic tumors were selected:

- patients without recurrence with a 5-year minimal follow-up;

- patients with recurrences: to the regional lymph nodes (stage III) or distant sites (stage IV) or death from melanoma. We included only cases with regional metastasis (stage III) or any type of distant metastasis (stage IV). Cases with microscopic metastatic lymph node deposits (micrometastasis) were excluded, as their clinical relevance is not fully resolved. For patients with in-transit cutaneous metastasis (stage III), we checked that the initial re-excision was complete and that the cutaneous metastasis was distant from the initial scar to avoid inclusion of a local cutaneous recurrence.

Homogeneity in data recording between centers was reached using a detailed form for each patient.

\section{Histopathological Review}

An independent triple-blind histopathological review of all cases was performed on one hematoxylin-eosin-saffron (HES)-stained slide of each case by three expert French pathologists (CB, AF and BV) without the knowledge of initial diagnosis, followup or FISH results. The three pathologists had to specify the degree of ambiguousness $(0=$ non-ambiguous; $1=$ ambiguous and $2=$ very ambiguous). Each pathologist had finally to classify the lesion either as ambiguous melanocytic lesion 'favor 
benign' (A-) or as 'favor malignant' $(\mathrm{A}+$ ). Finally, a case was considered as 'favor benign' $(A-)$ if the three pathologists agreed $(\mathrm{A}-\mathrm{A}-\mathrm{A}-)$ and 'favor malignant' $(\mathrm{A}+)$ if at least two pathologists diagnosed the tumor as $\mathrm{A}+$ (because we favored the diagnosis of malignancy and not of discordance with two $\mathrm{A}+$ ). If only one out of three pathologists disagreed $(\mathrm{A}+\mathrm{A}-\mathrm{A}-)$, the case was considered as discordant (D).

\section{FISH Analysis}

Areas of interest for FISH analysis were selected on HES-stained slides by a single pathologist (BV).

A four-color Vysis Melanoma FISH Probe kit from Abbott Molecular Inc. (Abbott France, Rungis, France) was used. This set of probes targets 6p25 (RREB1: Ras-responsive element-binding protein-1), 6q23 (MYB: myeloblastosis), 11q13 (CCDN1: cyclin D1) and chromosome 6 centromere (CEP6). They are labeled, respectively, with Spectrum Red, Spectrum Gold, Spectrum Green and Spectrum Aqua. FISH was performed on 5- $\mu \mathrm{m}$ sections of formalin-fixed paraffin-embedded tissues according to the protocol provided by Abbott Molecular Inc. Briefly, sections were mounted on SuperFrost Plus positively charged slides, baked at $56^{\circ} \mathrm{C}$ overnight, deparaffinized, submersed in $1 \times \mathrm{SSC}, \mathrm{pH} 6.3$, at $80^{\circ} \mathrm{C}$ for $35 \mathrm{~min}$ and washed in distilled water for $3 \mathrm{~min}$. After protease digestion (4 mg pepsin per $\mathrm{ml}$ in $0.2 \mathrm{~N}$ $\mathrm{HCl})$ at $37^{\circ} \mathrm{C}$ for $15 \mathrm{~min}$, sections were rinsed in distilled water for $3 \mathrm{~min}$, passed through graded ethanol and air-dried. Sections were co-denaturated with probe at $73^{\circ} \mathrm{C}$ for $5 \mathrm{~min}$ and hybridized at $37^{\circ} \mathrm{C}$ overnight using an automated ThermoBrite codenaturation oven (Molecular Abbott Inc.). Next, slides were placed in washing buffer $(2 \times$ SSC/ $0.3 \%$ NP40; Abbott Molecular Inc.) at room temperature for $2-10 \mathrm{~min}$ to remove the coverslips and then immersed in $73^{\circ} \mathrm{C}$ washing buffer for $2 \mathrm{~min}$, air-dried and mounted with Vectashield mounting medium with DAPI (Vector laboratories, Burlingame, CA, USA). The slides were analyzed with an epifluorescence microscope equipped with appropriate single band-pass filters (Abbott Molecular Inc.). Tumorbearing areas were selected using DAPI filter at low magnification ( $\times 20$ objective), and then evaluated for the presence of nuclei-harboring abnormal copy numbers of any probe. FISH analysis was performed by a trained cytogenetics specialist (MC) independently of the diagnosis and follow-up of patients. The slide was considered as interpretable if the following requirements were fulfilled: (1) signals bright, distinct and easily evaluable; (2) background relatively free of fluorescent particles or haziness; and (3) nuclei not overlapping to avoid false counting. For unsatisfactory cases, the FISH procedure on fresh slides was repeated three times with some modifications before considering the case as uninterpretable.
The entire section was examined to visually select clusters with an abnormal profile, and areas of interest selected by the pathologist were especially inspected. Three abnormal areas with the most significant copy number changes were selected for counting. Thirty nuclei were analyzed under high power $(\times 100)$ objective. The criteria for FISH positivity were provided recently by Gerami et al: ${ }^{10}$ (A) gain in RREB1 relative to CEP6 greater than $55 \%$; (B) gain in RREB1 greater than $29 \%$; (C) loss of $M Y B$ relative to CEP 6 greater than $40 \%$ and (D) gain in $C C D N 1$ greater than $38 \%$. The cases were considered as polyploid when all signals were simultaneously greater than 2 in the majority of nuclei examined.

\section{Statistical Analysis}

For non-equivocal melanocytic tumors, the sensitivity of the FISH assay was determined using histopathological diagnosis as a gold standard. For ambiguous melanocytic tumors, sensitivity of the assay was determined first using patients' outcome and then histopathological diagnosis of the three pathologists as a gold standard. Sensitivity of histopathological review was determined using outcome. SAS version 8.2 , on a UNIX operating system, was used to analyze the data. Kaplan-Meier curves were constructed using Proc LIFETEST in SAS. A multivariate survival analysis with a Cox regression model was used to determine the impact of age (under 17 years, range 17-59 years, > $>59$ years), location (axial vs extremities), FISH result and histopathological expert diagnosis, respectively. The hazard ratio of the FISH status effect on metastasis from the unadjusted Cox regression model was estimated using Proc PHREG.

\section{Results}

\section{FISH Results in Non-equivocal Melanomas and Nevi}

Among the 43 cases, only four were found uninterpretable by FISH according to our guidelines (efficiency 91\%), probably due to the fixation procedure. Seventeen out of 19 nevi (89.5\%) were negative (Table 1). The two FISH-positive nevi cases were positive for only one criterion: RREB1 gain for one (consequence of chromosome 6 trisomy) and $M Y B$ deletion for the other (Figure 1). Seventeen out of 20 melanomas (85\%) were FISH positive. Similarly, four out of 17 melanomas were positive for only one criterion, whereas nine (53\%) and four $(23.5 \%)$ malignant cases were positive for two and three criteria, respectively. The most frequent criteria found positive were RREB1 gain (12/17, $71 \%$ ), followed by $M Y B$ deletion (11/17, 65\%). Both acral lentiginous melanoma cases presented the same profile with $C C D N 1$ gain and $M Y B$ deletion. For the other melanoma subtypes, there were several 
Table 1 Data of the 39 non-equivocal melanomas and nevi

\begin{tabular}{|c|c|c|c|c|c|c|}
\hline Case & Age & Sex & Site & Subtype & $\begin{array}{c}\text { Breslow's } \\
\text { depth }(\mathrm{mm})\end{array}$ & $\begin{array}{l}\text { FISH } \\
\text { result }\end{array}$ \\
\hline \multicolumn{7}{|c|}{ Melanomas } \\
\hline 1 & 69 & $\mathrm{~F}$ & Scalp & SSM & 1.2 & - \\
\hline 2 & 48 & $\mathrm{~F}$ & Trunk & SSM & 1.2 & - \\
\hline 3 & 85 & $\mathrm{M}$ & Thigh & SSM & 2 & - \\
\hline 4 & 65 & $\mathrm{M}$ & Shoulder & SSM & 1.85 & $t^{\mathrm{B}, \mathrm{D}}$ \\
\hline 5 & 48 & $\mathrm{M}$ & Trunk & SSM & 15 & $t^{\mathrm{B}, \mathrm{D}}$ \\
\hline 6 & 77 & $\mathrm{M}$ & $?$ & SSM & 2.15 & $t^{\mathrm{A}, \mathrm{B}}$ \\
\hline 7 & 96 & $\mathrm{M}$ & Shoulder & SSM & 1 & $t^{\mathrm{B}, \mathrm{C}}$ \\
\hline 8 & 82 & $\mathrm{M}$ & Trunk & SSM & 1.8 & $+^{\mathrm{C}}$ \\
\hline 9 & 72 & $\mathrm{M}$ & Face & SSM & 3.6 & $+^{\mathrm{C}}$ \\
\hline 10 & 70 & $\mathrm{M}$ & Arm & SSM & 1.32 & $t^{\mathrm{B}, \mathrm{D}}$ \\
\hline 11 & 74 & $\mathrm{M}$ & Neck & LMM & 4 & $+^{\mathrm{B}, \mathrm{C}}$ \\
\hline 12 & 69 & $\mathrm{~F}$ & Face & LMM & 0.2 & $t^{\mathrm{B}, \mathrm{D}}$ \\
\hline 13 & 85 & $\mathrm{M}$ & Face & LMM & 4.7 & $+^{\mathrm{A}, \mathrm{B}, \mathrm{C}}$ \\
\hline 14 & 86 & $\mathrm{~F}$ & Acral & ALM & 0.58 & $+^{\mathrm{C}, \mathrm{D}}$ \\
\hline 15 & 62 & $\mathrm{M}$ & Acral & ALM & 1.8 & $++^{\mathrm{C}, \mathrm{D}}$ \\
\hline 16 & 89 & $\mathrm{~F}$ & Arm & Unclassifiable & 7.41 & $+{ }^{\mathrm{A}, \mathrm{B}, \mathrm{C}}$ \\
\hline 17 & 40 & $\mathrm{~F}$ & Leg & Unclassifiable & 26 & $+^{\mathrm{A}, \mathrm{B}, \mathrm{C}}$ \\
\hline 18 & 74 & $\mathrm{M}$ & Scalp & Unclassifiable & 7 & $+^{\mathrm{C}}$ \\
\hline 19 & 73 & $\mathrm{M}$ & Arm & Unclassifiable & 5.7 & $+^{\mathrm{B}, \mathrm{C}, \mathrm{D}}$ \\
\hline 20 & 60 & $\mathrm{~F}$ & Scalp & Desmoplastic & 7 & $+^{\mathrm{B}}$ \\
\hline \multicolumn{7}{|l|}{ Nevi } \\
\hline 1 & 50 & $\mathrm{M}$ & Trunk & Junctional & & - \\
\hline 2 & 5 & $\mathrm{~F}$ & Scalp & Compound & & $t^{\mathrm{C}}$ \\
\hline 3 & 15 & $\mathrm{~F}$ & Finger & Compound & & - \\
\hline 4 & 34 & $\mathrm{M}$ & Trunk & Compound & & - \\
\hline 5 & 72 & $\mathrm{M}$ & Trunk & Compound & & $t^{\mathrm{B}}$ \\
\hline 6 & 49 & $\mathrm{~F}$ & Chin & Dermal & & - \\
\hline 7 & 65 & $\mathrm{M}$ & Foot & Dermal & & - \\
\hline 8 & 66 & $\mathrm{M}$ & Face & Dermal & & - \\
\hline 9 & 29 & $\mathrm{M}$ & Neck & Dermal & & - \\
\hline 10 & 7 & $\mathrm{M}$ & Buttock & Congenital & & - \\
\hline 11 & 4 & $\mathrm{M}$ & Knee & Congenital & & - \\
\hline 12 & 47 & $\mathrm{M}$ & Trunk & Congenital & & - \\
\hline 13 & 22 & $\mathrm{M}$ & Trunk & Congenital & & - \\
\hline 14 & 28 & $\mathrm{M}$ & Trunk & Blue nevus & & - \\
\hline 15 & 36 & $\mathrm{~F}$ & Leg & Blue nevus & & - \\
\hline 16 & 3 & $\mathrm{~F}$ & Leg & Spitz-Reed & & - \\
\hline 17 & 13 & $\mathrm{M}$ & Thigh & Spitz-Reed & & - \\
\hline 18 & 43 & $\mathrm{M}$ & Thigh & Spitz-Reed & & - \\
\hline 19 & 18 & $\mathrm{~F}$ & Vulvar & Genital type & & - \\
\hline
\end{tabular}

Abbreviations: ALM: acral lentiginous melanoma; LMM: lentiginous malignant melanoma; SSM: superficial spreading melanoma. FISH result: negative (-); positive (+): $\mathrm{A}=R R E B 1 / C E P 6$ gain; $\mathrm{B}=R R E B 1$ gain; $\mathrm{C}=M Y B / C E P 6$ loss; and $\mathrm{D}=C C D N 1$ gain

positive criteria. Among melanomas, the three FISHnegative cases were superficial spreading melanoma thinner than $2 \mathrm{~mm}$ (Table 1). Altogether, in these non-equivocal melanocytic lesions, we achieved sensitivity $85 \%$, specificity $90 \%$, positive predictive value $89.5 \%$ and negative predictive value $86 \%$.

\section{FISH Results in the Ambiguous Melanocytic Tumors}

Eighteen out of 113 cases initially selected were excluded owing to incomplete follow-up data or less than 5 years recurrence-free follow-up (eight cases), micrometastasis (three cases), doubt between cutaneous recurrence and in-transit cutaneous metastasis (three cases), inappropriate fixative such as Bouin's fluid (three cases) and insufficient number of melanocytic cells on new sections (one case). Ninety out of 95 were interpretable (efficiency 95\%). Out of 90 ambiguous melanocytic lesions, 67 cases $(74.5 \%)$ were determined as FISH negative and 23 cases $(25.5 \%)$ as FISH-positive (Table 2). Among the 23 FISH-positive cases, there were five cases with three positive criteria, 11 cases with two criteria and seven cases with one criterion.

\section{Clinical and follow-up data}

Fifty-two women and 38 men were included (sex ratio: 1.4), with a mean age of 33 years (range: $4-73$ years). Nine cases concerned pre-pubescent children (under 11 years). Sixty-nine out of 90 patients $(77 \%)$ had a recurrence-free follow-up of at least 5 years, with a mean follow-up of 9 years (range: 5-19 years). Twenty-one patients $(23 \%)$ exhibited 
a

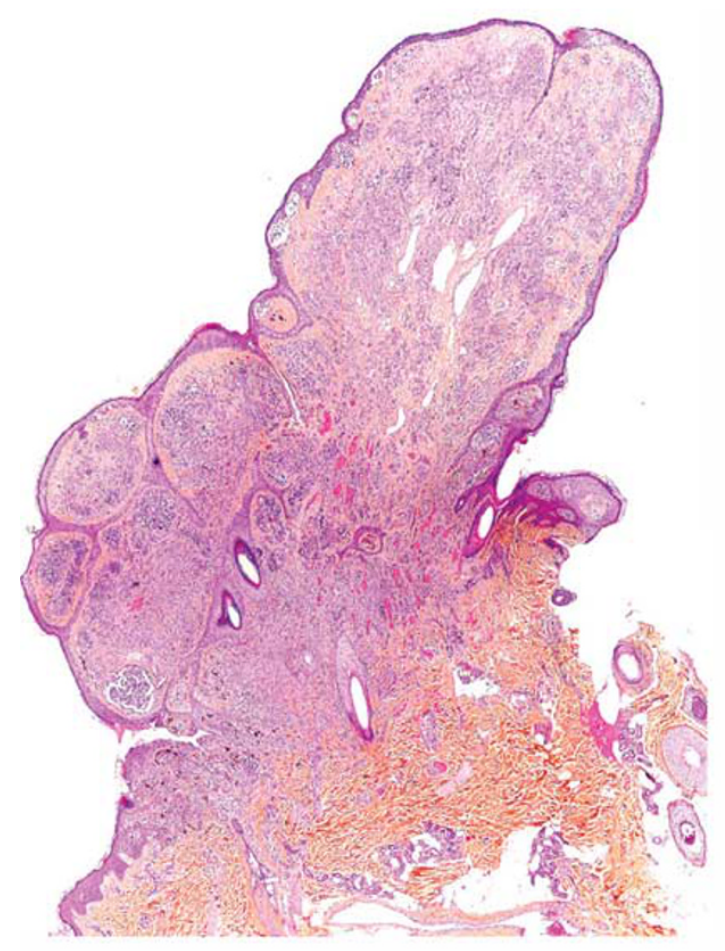

b
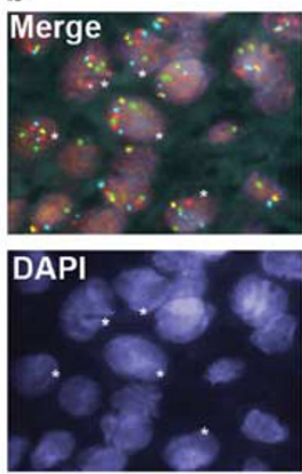

C
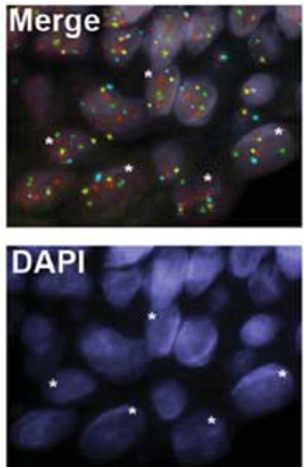
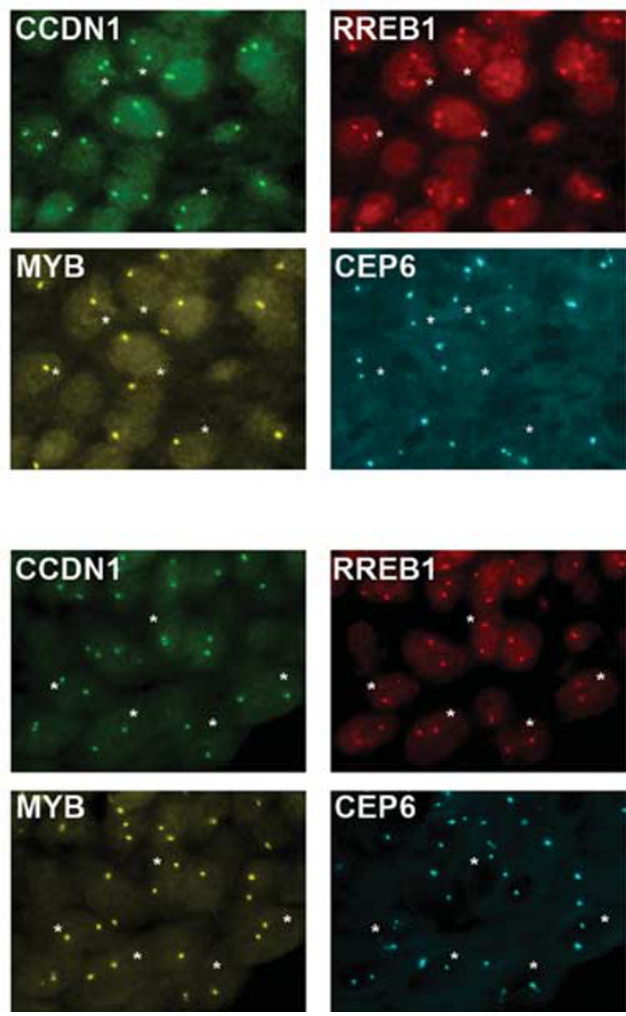
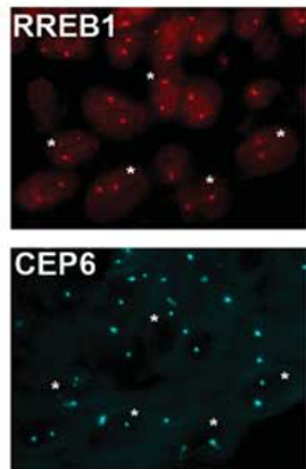

Figure 1 Example of fluorescence in situ hybridization (FISH)-positive nevus. (a) Compound nevus without atypia (hematoxylin-eosinsaffron $(H E S) \times 100)$. (b) FISH-positive result in the dermal component: cyclin D1 (CCDN1)/Ras-responsive element-binding protein-1 (RREB1)/myeloblastosis (MYB)/chromosome 6 centromere (CEP6) signals: 2/2/1/2 (*nuclei showing MYB deletion). (c) FISH-negative result in the normal epidermis (internal negative control): CCDN1/RREB1/MYB/CEP6 signals: 2/2/2/2 (*nuclei showing normal profile).

regional (stage $\mathrm{III}=12$ cases) or distant metastases (stage IV = 9 cases; lung, liver, bone, spleen, regional parotid or brain metastases). Among patients with stage III, 10 had lymph node metastases and two in-transit cutaneous metastases. Mean follow-up of patients with a poor course was 4 years (range: 6 months-20 years).

\section{Histopathological review}

The ambiguity of the lesion as determined by the first dermatopathologist was confirmed by the panel of three reviewers in all cases. These ambiguous lesions were classified mostly as spitzoid tumors (45/90 cases, 50\%), deep penetrating tumors (atypical or raising the question of melanoma simulating a deep penetrating naevus, 10 cases), nevoid melanoma (eight cases), cellular blue nevus or melanomasimulating cellular blue nevus (seven cases), superficial spreading melanoma with radial growth phase (five cases) or vertical growth phase (three cases), atypical acral tumor (three cases) and other types of atypical nevus (nine cases). The majority of ambiguous melanocytic tumors $(49 / 90,54 \%)$ were diagnosed as 'favor malignant' $(\mathrm{A}+)$ by the three pathologists (41/49 cases) or by two of the three pathologists (8/49 cases). Thirty-two (32/90, 36\%) were diagnosed as 'favor benign' $(\mathrm{A}-$ ) and nine
$(10 \%)$ had a discordant diagnosis (D). These nine discordant cases were recurrence free over a 5-year follow-up. Twenty out of 49 patients (41\%) diagnosed as 'favor malignant' presented metastases. Among the 32 tumors classified as 'favor benign', all had a 5-year recurrence-free follow-up, except one patient with a histological diagnosis of cellular blue nevus presenting with lung metastases. By comparison with outcome, the sensitivity and specificity of histopathological review were, respectively, 95 and $52 \%$. Positive and negative predictive values were 41 and $97 \%$.

\section{Comparison of FISH results with outcome}

Fifty-five out of 69 cases (80\%) with 5-year recurrence-free follow-up were FISH negative for all criteria and $14(20 \%)$ were FISH positive for at least one criterion (five cases for one criterion, seven cases for two criteria and two cases for three criteria). Among 21 patients with a secondary tumoral event, nine (43\%) were FISH positive (two cases for one criterion, four cases for two criteria and three cases for three criteria). The number of abnormalities was not proportional to the course of the disease. By comparison with outcome, the sensitivity and specificity of this FISH analysis were, respectively, 43 and $80 \%$; positive predictive 
Table 2 Characteristics of ambiguous melanocytic tumors

\begin{tabular}{|c|c|c|c|c|c|c|}
\hline $\begin{array}{l}\text { Type of ambiguous } \\
\text { tumor }\end{array}$ & Case no. & $A g e^{\mathrm{a}}$ & Sex & $\begin{array}{c}\text { Expert } \\
\text { diagnosis }\end{array}$ & $F I S H^{\mathrm{c}}$ & Adverse event \\
\hline \multirow[t]{45}{*}{ Spitz tumor } & 2 & 31 & $\mathrm{~F}$ & $\mathrm{~A}+$ & - & - \\
\hline & 5 & 20 & $\mathrm{~F}$ & $\mathrm{~A}+$ & - & - \\
\hline & 12 & 28 & $\mathrm{~F}$ & $\mathrm{~A}+$ & - & - \\
\hline & 78 & 42 & $\mathrm{M}$ & $\mathrm{A}+$ & - & - \\
\hline & 82 & 36 & M & $\mathrm{A}+$ & - & - \\
\hline & 89 & 34 & $\mathrm{~F}$ & $\mathrm{~A}+$ & - & - \\
\hline & 92 & 8 & $\mathrm{~F}$ & $\mathrm{~A}+$ & - & - \\
\hline & 93 & 32 & $\mathrm{M}$ & $\mathrm{A}+$ & - & - \\
\hline & 99 & 26 & $\mathrm{~F}$ & $\mathrm{~A}+$ & - & - \\
\hline & 101 & 42 & $\mathrm{~F}$ & $\mathrm{~A}+$ & - & - \\
\hline & 102 & 20 & $\mathrm{~F}$ & $\mathrm{~A}+$ & - & - \\
\hline & 15 & 18 & $\mathrm{~F}$ & $\mathrm{~A}+$ & - & Cutaneous/lung/liver M \\
\hline & 52 & 14 & M & $\mathrm{A}+$ & - & Subcutaneous M (+LN micro-M) \\
\hline & 53 & 16 & $\mathrm{M}$ & $\mathrm{A}+$ & - & Subcutaneous/peritoneal/brain M \\
\hline & 64 & 6 & $\mathrm{~F}$ & $\mathrm{~A}+$ & - & Lymph node $\mathrm{M}$ \\
\hline & 67 & 56 & $\mathrm{~F}$ & $\mathrm{~A}+$ & - & $\begin{array}{l}\text { Lymph node/parotid/pancreas/liver/bone/spleen } \\
\text { M (dead from melanoma) }\end{array}$ \\
\hline & 6 & 70 & M & $\mathrm{A}+$ & $t^{\mathrm{A}, \mathrm{B}, \mathrm{C}}$ & - \\
\hline & 59 & 51 & $\mathrm{~F}$ & $\mathrm{~A}+$ & $+^{\mathrm{C}}$ & - \\
\hline & 88 & 20 & M & $\mathrm{A}+$ & $++^{\mathrm{B}, \mathrm{D}}$ & - \\
\hline & 91 & 6 & $\mathrm{~F}$ & $\mathrm{~A}+$ & $+t^{\mathrm{B}, \mathrm{D}}$ & - \\
\hline & 95 & 20 & $\mathrm{~F}$ & $\mathrm{~A}+$ & $+t^{\mathrm{B}, \mathrm{D}}$ & - \\
\hline & 97 & 33 & $\mathrm{M}$ & $\mathrm{A}+$ & $++^{\mathrm{B}, \mathrm{D}}$ & - \\
\hline & 10 & 20 & $\mathrm{~F}$ & $\mathrm{~A}+$ & $+^{\mathrm{D}}$ & Lymph node $\mathrm{M}$ \\
\hline & 13 & 28 & $\mathrm{~F}$ & $\mathrm{~A}+$ & $+^{\mathrm{A}, \mathrm{B}}$ & Lymph node $\mathrm{M}$ \\
\hline & 37 & 56 & M & $\mathrm{A}+$ & $+^{\mathrm{B}, \mathrm{D}}$ & Lymph node M (dead from melanoma) \\
\hline & 51 & 23 & $\mathrm{~F}$ & $\mathrm{~A}+$ & $++^{\mathrm{B}, \mathrm{D}}$ & Lymph node $\mathrm{M}$ \\
\hline & 56 & 12 & M & $\mathrm{A}+$ & $+^{\mathrm{B}, \mathrm{C}, \mathrm{D}}$ & Subcutaneous M \\
\hline & 107 & 42 & $\mathrm{~F}$ & $\mathrm{~A}+$ & $+^{\mathrm{C}}$ & Lymph node/lung/liver M \\
\hline & 11 & 21 & M & A- & - & - \\
\hline & 21 & 53 & M & $A-$ & - & - \\
\hline & 34 & 14 & M & $\mathrm{A}-$ & - & - \\
\hline & 38 & 4 & M & $A-$ & - & - \\
\hline & 48 & 20 & $\mathrm{~F}$ & A- & - & - \\
\hline & 49 & 46 & $\mathrm{~F}$ & $\mathrm{~A}-$ & - & - \\
\hline & 76 & 44 & $\mathrm{~F}$ & A- & - & - \\
\hline & 96 & 6 & M & A- & - & - \\
\hline & 103 & 44 & $\mathrm{~F}$ & $\mathrm{~A}-$ & - & - \\
\hline & 105 & 11 & $\mathrm{~F}$ & A- & - & - \\
\hline & 108 & 33 & M & A- & - & - \\
\hline & 110 & 17 & $\mathrm{~F}$ & A- & - & - \\
\hline & 114 & 24 & $\mathrm{~F}$ & A- & - & - \\
\hline & 87 & 13 & $\mathrm{~F}$ & $\mathrm{~A}-$ & $t^{\mathrm{D}}$ & - \\
\hline & 104 & 23 & M & $\mathrm{D}$ & - & - \\
\hline & 40 & 37 & $\mathrm{~F}$ & $\mathrm{D}$ & $+^{\mathrm{A}, \mathrm{B}}$ & - \\
\hline & 47 & 23 & M & $\mathrm{D}$ & $+^{\mathrm{C}}$ & - \\
\hline \multirow[t]{10}{*}{ DPN } & 57 & 43 & M & $\mathrm{A}+$ & - & Lymph node M \\
\hline & 58 & 28 & $\mathrm{~F}$ & $\mathrm{~A}+$ & - & Lymph node/liver M \\
\hline & 83 & 43 & M & $\mathrm{A}+$ & - & Lymph node $\mathrm{M}$ \\
\hline & 1 & 10 & $\mathrm{~F}$ & A- & - & - \\
\hline & 69 & 30 & M & A- & - & - \\
\hline & 86 & 20 & $\mathrm{~F}$ & A- & - & - \\
\hline & 106 & 7 & M & $\mathrm{A}-$ & - & - \\
\hline & 72 & 64 & $\mathrm{~F}$ & $\mathrm{D}$ & - & - \\
\hline & 19 & 12 & M & $\mathrm{D}$ & - & - \\
\hline & 71 & 44 & $\mathrm{~F}$ & $\mathrm{D}$ & - & - \\
\hline \multirow[t]{7}{*}{ CBN } & 68 & 67 & M & $\mathrm{A}+$ & $+^{\mathrm{A}, \mathrm{B}, \mathrm{C}}$ & Lymph node M (dead) \\
\hline & 25 & 71 & $\mathrm{~F}$ & A- & - & - \\
\hline & 27 & 19 & $\mathrm{~F}$ & A- & - & - \\
\hline & 54 & 53 & M & $\mathrm{A}-$ & - & Lymph node /lung M \\
\hline & 45 & 26 & M & A- & $t^{\mathrm{B}}$ & - \\
\hline & 70 & 5 & M & A- & - & - \\
\hline & 26 & 55 & M & $\mathrm{D}$ & - & - \\
\hline \multirow[t]{4}{*}{ Nevoid melanoma } & 17 & 73 & $\mathrm{~F}$ & $\mathrm{~A}+$ & - & - \\
\hline & 16 & 41 & $\mathrm{~F}$ & $\mathrm{~A}+$ & - & Lymph node M \\
\hline & 20 & 37 & $\mathrm{~F}$ & $\mathrm{~A}+$ & - & - \\
\hline & 63 & 67 & $\mathrm{M}$ & $\mathrm{A}+$ & $+^{\mathrm{A}, \mathrm{B}}$ & - \\
\hline
\end{tabular}


Table 2 Continued

\begin{tabular}{|c|c|c|c|c|c|c|}
\hline $\begin{array}{l}\text { Type of ambiguous } \\
\text { tumor }\end{array}$ & Case no. & $A g e^{\mathrm{a}}$ & Sex & $\begin{array}{c}\text { Expert } \\
\text { diagnosis }^{\mathrm{b}}\end{array}$ & FISH $^{\mathrm{c}}$ & Adverse event \\
\hline & 66 & 56 & M & $A+$ & $++^{\mathrm{B}, \mathrm{C}, \mathrm{D}}$ & Bone M \\
\hline & 84 & 41 & $\mathrm{~F}$ & $A+$ & $+^{\mathrm{B}, \mathrm{D}}$ & - \\
\hline & 85 & 12 & $\mathrm{M}$ & $\mathrm{A}+$ & - & - \\
\hline & 109 & 50 & M & $A+$ & $+^{\mathrm{A}, \mathrm{B}, \mathrm{C}}$ & - \\
\hline \multicolumn{7}{|l|}{ SSM } \\
\hline RGP & 18 & 28 & $\mathrm{~F}$ & $\mathrm{~A}+$ & - & - \\
\hline RGP & 24 & 23 & $\mathrm{~F}$ & $A+$ & - & - \\
\hline RGP & 30 & 54 & $\mathrm{~F}$ & $A+$ & - & - \\
\hline RGP & 98 & 40 & $\mathrm{~F}$ & $\mathrm{~A}+$ & - & - \\
\hline RGP & 113 & 30 & $\mathrm{~F}$ & $A+$ & - & - \\
\hline VGP & 65 & 54 & M & $\mathrm{A}+$ & - & - \\
\hline VGP & 32 & 45 & $\mathrm{~F}$ & $\mathrm{~A}+$ & - & Lymph node M (dead) \\
\hline VGP & 4 & 44 & $\mathrm{~F}$ & $A+$ & - & Cutaneous/lymph node/lung/liver M \\
\hline \multicolumn{7}{|l|}{ Others } \\
\hline ALM & 31 & 54 & $\mathrm{~F}$ & $\mathrm{~A}+$ & $t^{\mathrm{B}, \mathrm{C}}$ & Lung M (dead) \\
\hline Acral Nevus & 41 & 6 & $\mathrm{M}$ & A- & - & - \\
\hline Acral Nevus & 23 & 28 & $\mathrm{~F}$ & A- & - & - \\
\hline Compound N & 79 & 36 & $\mathrm{~F}$ & A- & $t^{\mathrm{C}}$ & - \\
\hline Compound N & 44 & 46 & $\mathrm{~F}$ & $\mathrm{~A}-$ & - & - \\
\hline Junctional N & 112 & 25 & $\mathrm{~F}$ & $\mathrm{~A}-$ & - & - \\
\hline Combined $\mathrm{N}$ & 28 & 18 & $\mathrm{M}$ & A- & - & - \\
\hline Combined N & 42 & 28 & $\mathrm{~F}$ & A- & - & - \\
\hline Congenital N & 29 & 51 & $\mathrm{M}$ & A- & - & - \\
\hline Congenital N & 94 & 53 & $\mathrm{M}$ & A- & - & - \\
\hline Atypical N & 81 & 38 & $\mathrm{~F}$ & $\mathrm{D}$ & - & - \\
\hline Atypical N & 61 & 35 & $\mathrm{M}$ & $\mathrm{D}$ & - & - \\
\hline
\end{tabular}

Abbreviations: M: metastasis; DPN: deep penetrating nevus type; CBN: cellular blue nevus type tumor; SSM: superficial spreading melanoma; RGP: radial growth phase; VGP: vertical growth phase; ALM: acral lentiginous melanoma.

${ }^{\mathrm{a}}$ Age at diagnosis.

${ }^{b}$ Expert final histopathological diagnosis: favor malignant (A+); favor benign (A-) and discordant (D).

${ }^{\mathrm{C}}$ FISH result: positive (+), negative (-): $\mathrm{A}=R R E B 1 / C E P 6$ gain; $\mathrm{B}=R R E B 1$ gain; $\mathrm{C}=M Y B / C E P 6$ loss; and $\mathrm{D}=C C D N 1$ gain.

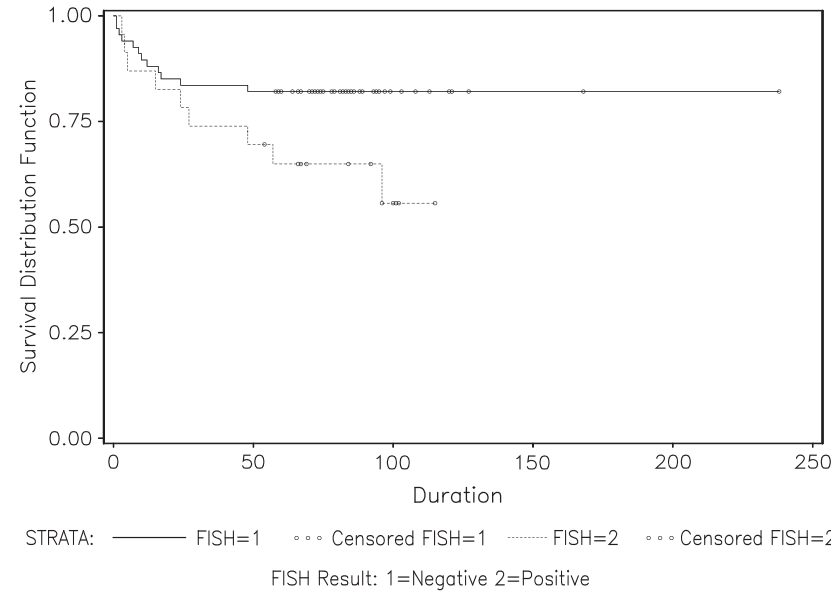

Figure 2 Kaplan-Meier curve-survival analysis (fluorescence in situ hybridization (FISH) result vs outcome).

value and negative predictive value were 39 and $82 \%$, respectively. Survival analysis (Kaplan-Meier method) showed a trend for a worse prognosis for FISH-positive patients (Figure 2), although this did not reach the statistical threshold $(P=0.07)$ in this small series. The sensitivity of FISH in the Spitz tumor group was higher than that in the overall cases $(54.5 \%)$.
Comparison of FISH results with histopathological diagnosis

In the 'favor benign' category (A-), $90 \%$ of cases (29/32) were FISH negative and only three cases were FISH positive with only one positive criterion (see Table 2). In the 'favor malignant' category of ambiguous cases, $37 \%$ of cases $(18 / 49)$ were FISH positive for at least one criterion (three cases for one criterion, 10 cases for two criteria and five cases for three criteria) (eg, see Figure 3). On the other hand, among 23 FISH-positive cases, 18 (78\%) were finally diagnosed as $\mathrm{A}+, 3 / 23(13 \%)$ as $\mathrm{A}-$ and $2 / 23(9 \%)$ as $\mathrm{D}$. The main positive criteria were RREB1 gain $(17 / 23,74 \%)$, followed by $C C D N 1$ gain $(11 / 23,48 \%)$ and $M Y B$ deletion (10/23, 43\%). Among 23 FISHpositive cases, nine cases $(39 \%)$ were polyploid. Interestingly, eight of these nine cases corresponded to Spitz tumors with seven 'favor malignant' and one 'favor benign'. One 'favor malignant' polyploid case was a nevoid melanoma (case 84). The only 'favor benign' FISH-positive Spitz tumor was not polyploid (case 87). Among discordant (D) cases, seven out of nine were FISH-negative cases and two were FISH-positive cases (see Table 2) and classified as Spitz tumors. Excluding discordant cases, sensitivity and specificity of FISH compared with histopathological diagnosis were, respectively, 36.7 and $90.6 \%$ (positive predictive value $86 \%$ and 

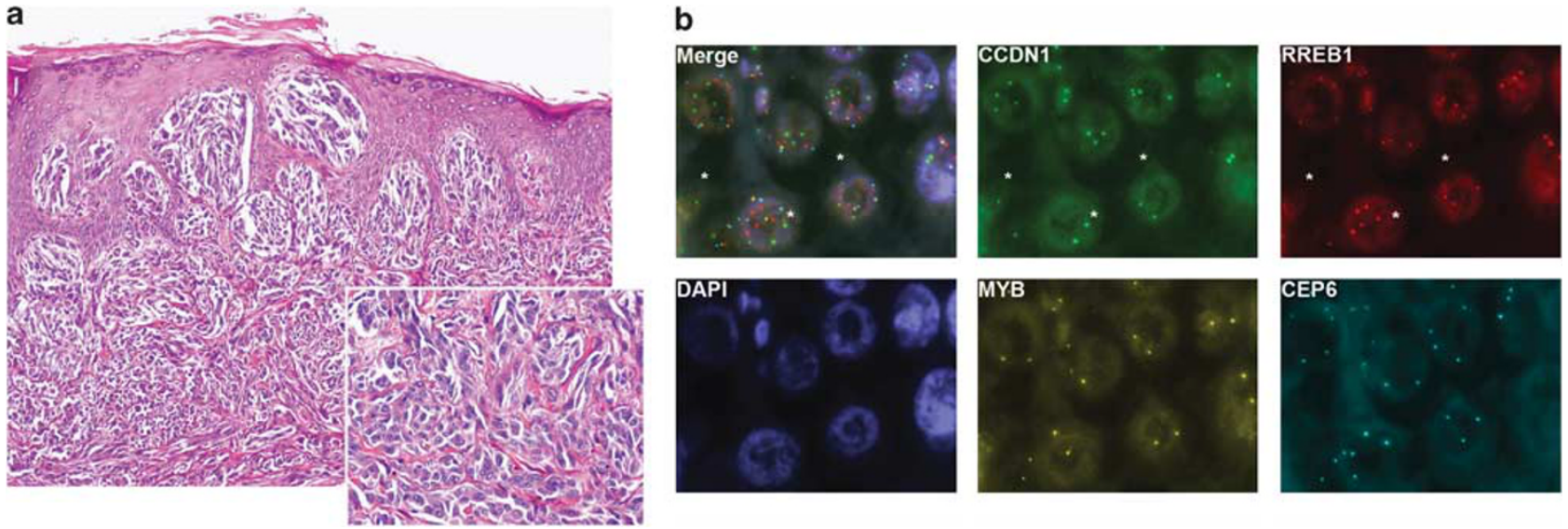

Figure 3 Example of fluorescence in situ hybridization (FISH)-positive ambiguous lesion (case 56): (a) hematoxylin-eosin-saffron (HES)stained section showing spitzoid melanocytic tumor with large intraepidermal nests of spindle and epithelioid cells $(\times 100)$. Inset: higher magnification $(\times 400)$. (b) FISH-positive result in the dermal component: *Nuclei showing Ras-responsive element-binding protein-1 (RREB1) and cyclin D1 (CCDN1) gain myeloblastosis (MYB) deletion.

Table 3 Multivariate Cox proportional hazard regression model of significant prognostic parameters

\begin{tabular}{|c|c|c|c|c|c|}
\hline & Standard error & $\chi^{2}$ & $\mathrm{P}$-value & Hazard ratio & $95 \% C I$ \\
\hline FISH & 0.46380 & 0.2812 & 0.5959 & 1.279 & $(0.515,3.174)$ \\
\hline Age group ${ }^{a}$ & 0.47056 & 2.0841 & 0.1488 & 0.507 & $(0.202,1.275)$ \\
\hline Expert diagnosis ${ }^{\mathrm{b}}$ & 1.03969 & 7.4261 & 0.0064 & 17.000 & $(2.216,130.45)$ \\
\hline Location $^{\mathrm{c}}$ & 0.46692 & 0.0210 & 0.8848 & 1.070 & $(0.428,2.672)$ \\
\hline
\end{tabular}

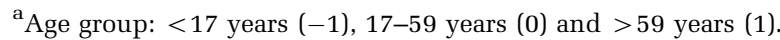

${ }^{\mathrm{b}}$ Expert diagnosis: malignant $(\mathrm{A}+)$ and benign $(\mathrm{A}-)$.

${ }^{\mathrm{C}}$ Location: extremities (0) and axial (1).

Bold values are significant result.
}

negative predictive value $48 \%$ ). Even if discordant cases were included as 'favor malignant' (as one pathologist thought it was malignant), sensitivity and specificity were, respectively, 34.5 and $91 \%$, with positive predictive value $87 \%$ and negative predictive value $43 \%$.

\section{Combination of FISH results and histopathological} diagnosis vs outcome

Interestingly, by combining the histopathological diagnosis with FISH results and by considering cases as truly malignant only if histopathological diagnosis was 'favor malignant' $(\mathrm{A}+)$ and FISH positive, the diagnosis was optimized, especially by increasing specificity $(76 \%$ instead of $52 \%$ for expert diagnosis alone), and also by improving sensitivity compared with FISH alone (90 vs 43\% for FISH result alone). Multivariate Cox regression showed that only expert diagnoses were statistically significant compared with metastases $P=0.0064$ (Table 3).

\section{Discussion}

This new FISH assay was first evaluated by Gerami et $a l^{10}$ on a cohort of 83 melanomas and 86 typical nevi, with a sensitivity of $86.7 \%$ and a specificity of $95.4 \%$. Their results were confirmed by the study of Morey et $a l^{12}$ on 10 metastases of melanomas, 10 primary melanomas and 10 nevi showing a sensitivity of $90 \%$ and a specificity of $95 \%$, and more recently by a new study by Gerami et $a l^{13}$ on 110 nevi and 123 melanoma (sensitivity $83 \%$ and specificity $94 \%$ ). Using the same updated threshold value on a series of 20 non-ambiguous melanomas and 19 nevi, we observed quite similar sensitivity of $85 \%$ and specificity of $90 \%$. In our study, two out of 19 nevi were FISH positive as well as 4/86 nevi in the study by Gerami and 1/10 nevi in Morey's study. Our two cases as well as Morey's nevus had only one positive criterion. No criterion other than histopathological review can be used to consider such cases as truly benign and therefore as 'false-positive' FISH data, as such lesions may have been cured by excision, so their spontaneous outcome is unknown. Such 'false-positive' cases emphasize the need to combine morphology and FISH data to achieve a final diagnosis, and underline the lack of interest in performing FISH for the diagnosis of typically benign or malignant cases. Concerning false-negative cases, our three FISH-negative melanomas were thin SSM (Breslow) between 1.2 and $2 \mathrm{~mm}$. However, the impact of thickness on FISH results was 
not shown by the study by Gerami et $a l .{ }^{10}$ A recent study proved the feasibility of this FISH test for in situ (very thin) melanocytic lesions in the so-called lentiginous junctional melanoma of the elderly. ${ }^{14}$ In this study, three out of 19 in situ melanomas were FISH negative. The main difficulty concerning melanocytic lesions is to define the gold standard for malignancy. It might be best to use histopathological diagnosis. Yet, diagnoses may differ between experts. ${ }^{4}$ It could also be to base judgment on metastatic evolution. Yet, well-excised melanomas may be cured and never metastasize. A particularly doubtful diagnosis for thin lesions has no impact on survival because complete excision leads to complete remission. On the other hand, the impact of a doubtful diagnosis in thick melanocytic lesions is great because treatment and follow-up are quite different. Therefore, our study shows that the histopathological diagnosis combined with FISH data could improve the accuracy of diagnosis.

In our study, the most frequent FISH-positive criterion was RREB1 gain (71\%), as observed by others. ${ }^{10,12,13}$ However in Morey's study, CCDN1 gain was as frequent as $R R E B 1$ gain in all type of melanomas $(70 \%){ }^{12}$ whereas we observed only $48 \%$ of $C C D N 1$ gain in FISH-positive cases. CCDN1 gain has been found associated with ALM and chronically sun-damaged skin melanoma subtypes. ${ }^{13,15}$ In our series, ALM cases shared the same chromosome aberration: CCDN1 gain and $M Y B$ deletion. This is in agreement with the genetic classification of melanomas based on sun exposure proposed by Bastian et al. ${ }^{11}$ To improve the diagnosis of acral melanoma or chronic sun-induced damage melanomas, the analysis of KIT mutations could provide ancillary data. ${ }^{16}$ Curtin et $a l^{16}$ found KIT mutations in 36\% of acral melanomas and $28 \%$ of chronic sun-induced damage melanomas, but never in melanoma on skin without chronic suninduced damage melanomas. The impact of FISH analysis of melanoma has to be determined not only for diagnostic purposes, but also for prognosis and treatment.

In ambiguous melanocytic tumors, such genetic analysis would primarily be very useful from a diagnostic point of view, as it would help pathologists to distinguish benign and malignant melanocytic lesions. Indeed, ambiguous lesions simulating a melanoma represent the most frequent cause of lawsuits for pathologists. ${ }^{9}$ Different genetic approaches have been used to facilitate this differential diagnosis. Koh et $a l^{17}$ proposed a DNA microarray-derived gene expression profiling study after microdissection on formalin-fixed and paraffin-embedded tissue sections of melanoma and nevus, including difficult melanocytic lesions. Thirty-six significant differentially expressed genes were identified. In comparison with nevi, melanomas expressed higher levels of genes promoting signal transduction, transcription and cell growth, but expression of L1CAM was reduced in melanomas relative to nevi. For this reason, gene signatures established using DNA microarray gene expression profiling could be useful for distinguishing melanomas from nevi as a supplement to standard histology. The reproducibility of such an approach has never been tested and its use in routine practice may not be as robust as FISH analysis.

Among ambiguous melanocytic tumors, the main group of problematic lesions is Spitz tumors (45/90 in our study) as frequently reported in the literature (eg, by Barnhill et $a l^{2}$ ). In our study, among 28 out of the 45 Spitz tumors diagnosed as 'favor malignant' $(\mathrm{A}+), 12$ were FISH positive and six of them underwent metastasis. Conversely, among the 16 FISH-negative 'favor malignant' Spitz cases, five also displayed metastases showing that histopathological analysis remains the gold standard for establishing the diagnosis of malignancy. A broader spectrum of FISH probes and further genetic analyses need to be evaluated in such FISH-negative patients with metastatic outcome. Among the 14 'favor benign' (A-) Spitz nevi, only one was FISH positive (CCDN1 gain) and was even recurrence free at 5-year follow-up. Two out of the three discordant Spitz cases were FISH positive without unfavorable course. Therefore, FISH positivity in ambiguous cases may strengthen the diagnosis of malignancy when at least one pathologist favors malignancy. In such 'favor malignant' Spitz cases, a FISH-negative result should not modify the expert interpretation. In 'favor benign' ambiguous cases, negative FISH data may lead to 'conservative' excision, as suggested by others. ${ }^{18}$ Using the same FISH melanoma kit, Gerami et $a l^{10}$ analyzed 27 ambiguous melanocytic tumors reviewed by two dermatopathologists also mostly represented by Spitz tumors (24/27). Six patients developed metastases and 21 remained free with at least a follow-up period of 5 years. FISH was positive in all six metastatic patients and in 6/21 non-metastatic patients. Among such Spitz tumors, a technical difficulty for interpretation is polyploidy. Eight of our nine polyploid cases corresponded to Spitz tumors (seven 'favor malignant' and one 'favor benign'). Interestingly, Isaac et $a l^{19}$ reported four out of 41 Spitz nevi as polyploidy that were confirmed by X-chromosome probe. The diseasefree survival times between FISH-positive and FISHnegative patients were significantly different $(P=0.003)$, as we observed in our study with a trend for better prognosis for FISH-negative patients $(P=0.07)$. This could be very useful in the future because prognosis of melanocytic lesions seems to be worse when the number of chromosomal anomalies increases. Therefore, FISH data associated with histopathological diagnosis could change the patient's management.

In our study, integration of FISH data along with histopathological diagnosis was found relevant for patient's management as the FISH result was found 
to improve the specificity of histopathological expert diagnosis alone $(76 \%$ instead of $52 \%)$. On the other hand, the sensitivity of the FISH test alone was also improved (90 vs 43\%). The most difficult issue for ambiguous melanocytic lesions is to determine what is the proof of malignancy between expert diagnosis or metastatic outcome. If expert diagnosis is taken to be the gold standard for ambiguous melanocytic lesions, the specificity of this FISH test is quite high (90.6\%). However between experts, the diagnostic reproducibility of ambiguous melanocytic tumors is quite low. ${ }^{4}$ On the other hand, if outcome is considered the most important criterion, FISH was found to provide good specificity (80\%). Even metastatic outcome is probably not so unfavorable in childhood and teenage Spitz tumors, as recently shown by Busam et al. ${ }^{20}$

Other applications of such a FISH melanoma kit have been reported. It could be useful for microstaging of melanoma, allowing the distinction between melanoma arising on nevus vs nevoid melanoma. ${ }^{21}$ It could also be useful if it really changes the treatment of patients, for example, with a thickness greater than 1 or $1.5 \mathrm{~mm}$ (according to the protocol), leading to interferon treatment or other adjuvant management such as sentinel node biopsy or adjuvant vaccine therapy. Other FISH applications have been reported to facilitate the differential diagnosis between (I) epithelioid blue nevus and cutaneous metastatic melanoma simulating blue nevus $^{22}$ or (ii) nevoid melanomas and mitotically active nevi, ${ }^{23}$ (iii) conjunctival nevi from melanomas $^{24}$ or (iv) intranodal nevus from metastatic melanoma. ${ }^{25}$

In conclusion, a single genetic test allowing the differential diagnosis between benign and malignant ambiguous melanocytic lesions does not yet exist. However, the practical value of the FISH test used in this study was that it reinforced the histopathological diagnosis of malignancy. In unequivocal melanomas it was highly sensitive. In ambiguous melanocytic tumors, especially in thick lesions, slides should first be submitted to a referring pathologist to improve the diagnosis and then a positive FISH test would strengthen the diagnosis of 'favor malignancy'. However, FISH testing did not formally identify patients at risk of metastasis. In unequivocal benign melanocytic ambiguous lesions, caution should be taken if the FISH test is positive, whereas a negative test would be an ancillary argument for benignity in 'favor benign' cases. ${ }^{18}$ In our opinion, FISH positivity alone without histopathological diagnosis of malignancy should not lead to modifying the patient's treatment. Finally, in cases with discordance between pathologists, the value of a positive FISH test was high when a single pathologist favored malignancy. Therefore, ambiguous lesions with doubtful or discordant histopathological analyses should be tested by FISH. In this group, FISH-positive cases should be managed in the same way as malignant and 'favor malignant' cases. Further genetic testing should be conducted on FISH-negative 'favor malignant' cases.

\section{Disclosure/conflict of interest}

The authors declare no conflict of interest.

\section{References}

1 vanDijk MC, Aben KK, van Hees F, et al. Expert review remains important in the histopathological diagnosis of cutaneous melanocytic lesions. Histopathology 2008;52:139-146.

2 Barnhill RL, Argenyi ZB, From L, et al. Atypical Spitz nevi/tumors: lack of consensus for diagnosis, discrimination from melanoma, and prediction of outcome. Hum Pathol 1999;30:513-520.

3 Cerroni L, Kerl H. Tutorial on melanocytic lesions. Am J Dermatopathol 2001;23:237-241.

4 Lodha S, Saggar S, Celebi JT, et al. Discordance in the histopathologic diagnosis of difficult melanocytic neoplasms in the clinical setting. J Cutan Pathol 2008;35:349-352.

5 Scolyer RA, Shaw HM, Thompson JF, et al. Interobserver reproducibility of histopathologic prognostic variables in primary cutaneous melanomas. Am J Surg Pathol 2003;27:1571-1576.

6 Cook MG, Clarke TJ, Humphreys S, et al. The evaluation of diagnostic and prognostic criteria and the terminology of thin cutaneous malignant melanoma by the CRC Melanoma Pathology Panel. Histopathology 1996;28:497-512.

7 Slater DN. Melanocytic intra-epidermal neoplasia (MIN). Histopathology 1997;30:195-197.

8 Elder DE, Xu X. The approach to the patient with a difficult melanocytic lesion. Pathology 2004;36: 428-434.

9 Massi G. Melanocytic nevi simulant of melanoma with medicolegal relevance. Virchows Arch 2007;451: 623-647.

10 Gerami P, Jewell SS, Morrison LE, et al. Fluorescence in situ hybridization (FISH) as an ancillary diagnostic tool in the diagnosis of melanoma. Am J Surg Pathol 2009;33:1146-1156.

11 Bastian BC, Olshen AB, LeBoit PE, et al. Classifying melanocytic tumors based on DNA copy number changes. Am J Pathol 2003;163:1765-1770.

12 Morey AL, Murali R, McCarthy SW, et al. Diagnosis of cutaneous melanocytic tumours by four-colour fluorescence in situ hybridisation. Pathology 2009;41: 383-387.

13 Gerami P, Mafee M, Lurtsbarapa T, et al. Sensitivity of fluorescence in situ hybridization for melanoma diagnosis using RREB1, MYB, Cep6, and 11q13 probes in melanoma subtypes. Arch Dermatol 2010;146: 273-278.

14 Newman MD, Mirzabeigi M, Gerami P. Chromosomal copy number changes supporting the classification of lentiginous junctional melanoma of the elderly as a subtype of melanoma. Mod Pathol 2009;22:1258-1262.

15 Curtin JA, Fridlyand J, Kageshita T, et al. Distinct sets of genetic alterations in melanoma. $\mathrm{N}$ Engl J Med 2005;353:2135-2147. 
16 Curtin JA, Busam K, Pinkel D, et al. Somatic activation of KIT in distinct subtypes of melanoma. J Clin Oncol 2006;24:4340-4346.

17 Koh SS, Opel ML, Wei JP, et al. Molecular classification of melanomas and nevi using gene expression microarray signatures and formalin-fixed and paraffinembedded tissue. Mod Pathol 2009;22:538-546.

18 McCalmont TH. Gone FISHing. J Cutan Pathol 2010;37:193-195.

19 Isaac AK, Lertsburapa T, Pathria Mundi J, et al. Polyploidy in Spitz nevi: a not uncommon karyotypic abnormality identifiable by fluorescence in situ hybridization. Am J Dermatopathol 2010;32:144-148.

20 Busam KJ, Murali R, Pulitzer M, et al. Atypical spitzoid melanocytic tumors with positive sentinel lymph nodes in children and teenagers, and comparison with histologically unambiguous and lethal melanomas. Am J Surg Pathol 2009;33:1386-1395.

21 Newman MD, Lertsburapa T, Mirzabeigi M, et al. Fluorescence in situ hybridization as a tool for microstaging in malignant melanoma. Mod Pathol 2009;22:989-995.

22 Pouryazdanparast $\mathrm{P}$, Newman $\mathrm{M}$, Mafee $\mathrm{M}$, et al. Distinguishing epithelioid blue nevus from blue nevus-like cutaneous melanoma metastasis using fluorescence in situ hybridization. Am J Surg Pathol 2009;33:1396-1400.

23 Gerami P, Wass A, Mafee M, et al. Fluorescence in situ hybridization for distinguishing nevoid melanomas from mitotically active nevi. Am J Surg Pathol 2009;33:1783-1788.

24 Busam KJ, Fang Y, Jhanwar SC, et al. Distinction of conjunctival melanocytic nevi from melanomas by fluorescence in situ hybridization. J Cutan Pathol 2010;37:196-203.

25 Dalton SR, Gerami P, Kolaitis NA, et al. Use of fluorescence in situ hybridization (FISH) to distinguish intranodal nevus from metastatic melanoma. Am J Surg Pathol 2010;34:231-237. 